\section{DMITSA: A simplified interrupted time-series analysis program}

\author{
JOHN CROSBIE \\ Deakin University, Victoria, Australia
}

and

\section{CHRISTOPHER F. SHARPLEY \\ Monash University, Victoria, Australia}

As has been discussed many times in the literature (e.g., Crosbie, 1987; Glass, Willson, \& Gottman, 1975; Gottman, 1981; Hartmann et al., 1980), it is invalid to employ a $t$ test or a similar statistical procedure with timeseries data, because such data are usually autocorrelated (i.e., there is a nonzero correlation between adjacent data points). If an observation is more similar to its predecessor than to the overall mean, the data have a positive autocorrelation. Conversely, if an observation is more dissimilar to its predecessor than to the overall mean, the data have a negative autocorrelation. Nonzero autocorrelation implies that some portion of a score carries over to the next score in the series. For example, a simple series (the first-order autoregressive) could be expressed algebraically in the following way:

$$
Y_{n}=X Y_{n-1}+e_{n},
$$

where $Y_{n}$ is the score at Time $n, X$ is the autocorrelation coefficient, $Y_{n-1}$ is the score at Time $n-1$, and $e_{n}$ is random error. This formula shows that the autocorrelation coefficient $(X)$ reflects the degree to which a score affects the next score in the series; if $X$ is close to 1 , each score is composed of nearly all of the previous score, but if $X$ is zero, a score has no influence on subsequent scores.

Conceptually, the purpose of interrupted time-series analysis (ITSA) is to remove autocorrelation so that statistical tests can be legitimately employed to determine whether there is a statistically significant change from Phase 1 to Phase 2 in the time series. There are two main ways to achieve this. One technique (used in the TMS program described by Glass et al., 1975) is to conduct an exhaustive search for the autocorrelation values that best fit the data, remove autocorrelation from each raw score to produce an uncorrelated series, then perform a $t$ test on the uncorrelated data to determine whether Phase 1 scores differ from Phase 2 scores. The other popular technique (used in the ITSE program described by Gottman, 1981, and Williams \& Gottman, 1982) employs matrix algebra to determine the autocorrelation values, changes in level, and changes in slope that best fit the data.

Reprints of this article are available from John Crosbie, Department of Psychology, Deakin University, VIC 3217, Australia.

\section{Techniques for Performing ITSA}

TMS. The TMS program assumes that the data have no slope, so, before TMS can be employed legitimately, any slope in the data must be removed (see Glass et al., 1975, and Gottman, 1981, for details of how to remove slope). As an example of why it is crucial to remove slope before using TMS, consider the data shown in Figure 1, which have a consistent slope across both phases, but no change from Phase 1 to Phase 2.

A $t$ test performed on these data would show that Phase 2 scores are significantly greater than Phase 1 scores (and removing autocorrelation would not alter this result); hence a treatment effect would be inferred, even though there was no real change. Therefore, if TMS were employed to analyze these data before the slope was removed, the results would erroneously suggest that there was a statistically significant change between the phases.

TMS tests many levels of autocorrelation (e.g., .02, $.04, .06$, etc.) to determine which of these provide the best fit for the data. If only one autocorrelation parameter is assumed (i.e., a score is affected only by the previous score in the series), TMS would usually test 100 levels of autocorrelation. If, however, three parameters are assumed (i.e., a score is affected by the three previous scores in the series), then $1,000,000$ tests would be required to obtain estimates of similar precision. This would be too time-consuming, so, when several autocorrelation parameters are assumed, TMS performs fewer tests, and consequently obtains less accurate estimates.

In summary, before TMS can be employed properly, some other procedure must be performed to remove any slope, and this complicates the task. Furthermore, the sequential search technique is laborious, time-consuming, and inefficient, and the resultant estimates of autocorrelation are less accurate than those obtained by the more efficient matrix algebra procedure.

ITSE. Unlike TMS, the ITSE program can be employed legitimately with slope in the data because ITSE

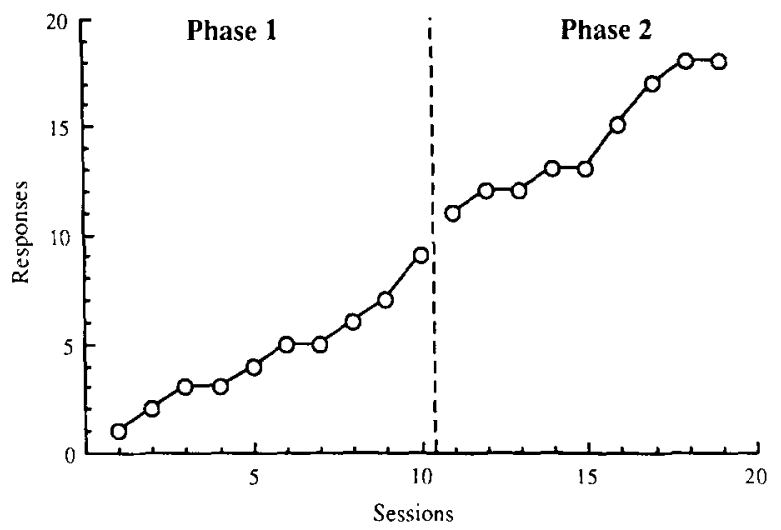

Figure 1. Hypothetical time-series data with no change in intercept or slope between the phases. 
DMITSA Version 1.0

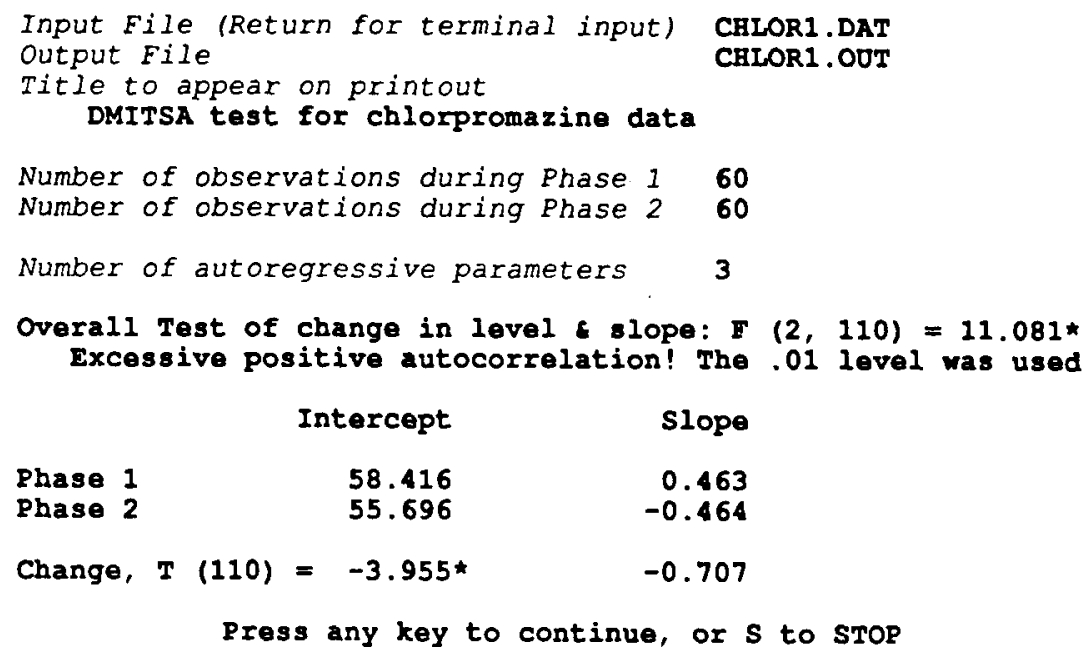

Figure 2. Program prompts (italics), user responses (bold), and screen output (bold) for a DMITSA analysis of CHLOR1.DAT.

models the data on the assumption that there is a slope. Matrix algebra is then employed to produce a line of best fit for each phase (after the influence of autocorrelation has been removed), and ITSE determines three things: (1) whether the Phase 2 line differs significantly in any way from the extension of the Phase 1 line, (2) whether these lines have significantly different intercepts on the vertical axis, and (3) whether these lines have significantly different slopes. With the data shown in Figure 1, ITSE would suggest that the phases do not differ significantly.

Compared with the TMS sequential search technique, the algebraic procedure employed by ITSE is faster, more efficient, and more accurate, and it can be performed satisfactorily with fewer data points. Therefore, in addition to the considerable advantage of being able to accommodate, and directly assess, slope in data, the technique employed by ITSE offers substantial benefits. For these reasons, this is the analytic procedure employed in DMITSA.

\section{Advantages of DMITSA}

Compared with TMS and ITSE, DMITSA has the following advantages: (1) it runs on IBM PC, XT, and AT computers and compatibles; (2) color is used to highlight program prompts and make results easier to read; (3) a data entry module is available within DMITSA, and the program can analyze data entered either at the terminal or via a file; (4) the output file can be named by the user during an analysis session (instead of employing a default name), which means that several analyses can be performed in a session without exiting the program to rename output files; (5) headings can be included on output files to provide better documentation of the data; (6) basic results are sent to the screen (for quick assessment, and to facilitate analysis of several data sets without leaving the program), and more comprehensive results are written to an optional output file; (7) all scores (in- stead of only the first and last score) are written to the output file to ensure that the analysis was performed on the correct data; (8) output is easier for nonexperts to understand; (9) data are read in freefield (i.e., their column locations in a file are irrelevant, provided each score is separated by at least one space or carriage return), so no complex FORTRAN format statement is required; (10) output is succinct (i.e., all output fits on one screen and one page of the output file); (11) an efficient matrix algebra technique is employed instead of the laborious sequential search procedure; (12) slope need not be removed from the data before analysis; (13) the model to be tested is easy to specify and requires no special expertise (i.e., the user merely specifies the number of autoregressive parameters to be assumed); (14) the significance of each result is computed with the appropriate degrees of freedom and critical values for the .05 level, then shown on the screen and written to the output file; and (15) warn-

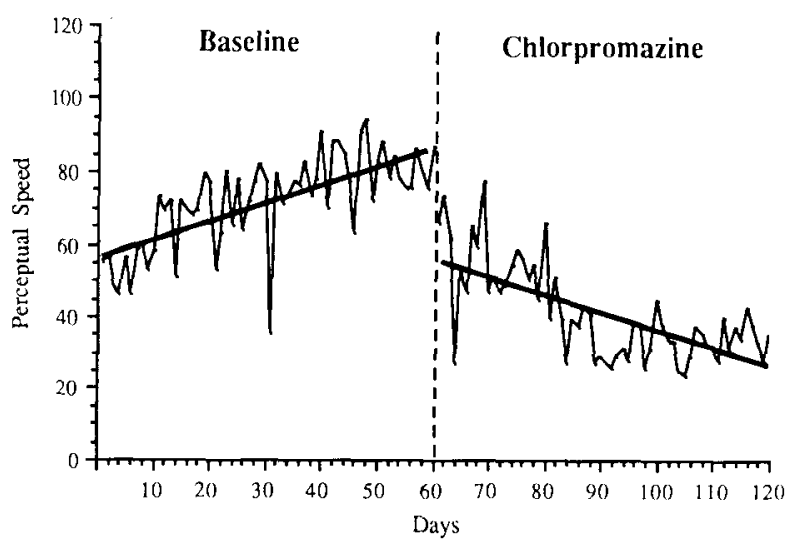

Figure 3. CHLOR1.DAT plus lines of best fit (thick solid lines) determined by DMITSA. 
ings are provided if the total amount of positive autocorrelation is so high that results could be misleading, and a more conservative alpha level (e.g., .01 instead of .05) is required to maintain the desired level of Type I error. Overall, DMITSA reflects the new age of statistical software for personal computers, and it represents a considerable advance beyond TMS and ITSE. In addition to providing a tutorial on ITSA in general, and DMITSA in particular, the manual also gives advice (based on our recent research) on how to minimize Type I and Type II error whenever DMITSA is employed.

\section{Input and Output}

Figure 2 shows the program prompts (in italics), responses that would be typed by the user (in bold), and screen output (in bold) for an analysis of the effects of chlorpromazine on perceptual speed (Glass et al., 1975, p. 21). In this example, the data are in a file called CHLOR1.DAT, and each score is separated by at least one space or carriage return. The output will be written to a file called CHLOR1.OUT, and will have "DMITSA test for chlorpromazine data" as a heading. There are 60 observations during Phase 1 (Baseline) and 60 observations during Phase 2 (Intervention), and DMITSA will assume that the data can be described adequately with three autoregressive parameters (i.e., each score is affected only by the previous three scores).

The next line shows the result of the omnibus test of any change in intercept or slope between the phases. In this example, the $F$ test suggests that there was some change, but warns that, because of excessive positive auto- correlation, the .01 alpha level was employed to maintain the probability of making a Type I error at the desired level of .05 . In this case, with 2 and 110 degrees of freedom, the $F$ of 11.081 is significant at the .01 level, and DMITSA shows this by printing "*" after the $F$ value. The next section of the screen output shows, for each phase, the intercept and slope of the line of best fit (i.e., steady-state least-squares solution; Gottman, 1981) after autocorrelation has been considered, plus a $t$ value associated with the change in intercept and change in slope. In the present example, the Phase 1 intercept is 58.416 and the Phase 2 intercept is 55.696, and this change is significant $[t(110)=-3.955, p<.01]$. In contrast, the change in slope from 0.463 to -0.464 is not significant $[t(110)=0.707$, n.s. $]$. Figure 3 shows the data employed in the present example, plus the lines of best fit (thick solid lines) for each phase. These results suggest that the intervention produced a significant reduction in intercept, but not in slope. The user can then continue analyzing these or other data by pressing any key except " $S$ ", which will terminate the program.

Figure 4 shows the output file (CHLOR1.OUT) generated by the above example. The heading ("DMITSA test for chlorpromazine data") is written on the first line, and all of the pre- and postintervention scores are written to the file so that the user can verify that the analysis was performed on the correct data. The program calculated three autoregressive parameters $(.131, .106$, and .076 , respectively), and each is displayed with a $t$ value for the null hypothesis that the autoregressive parameter is zero. The next section shows the same information that was dis-

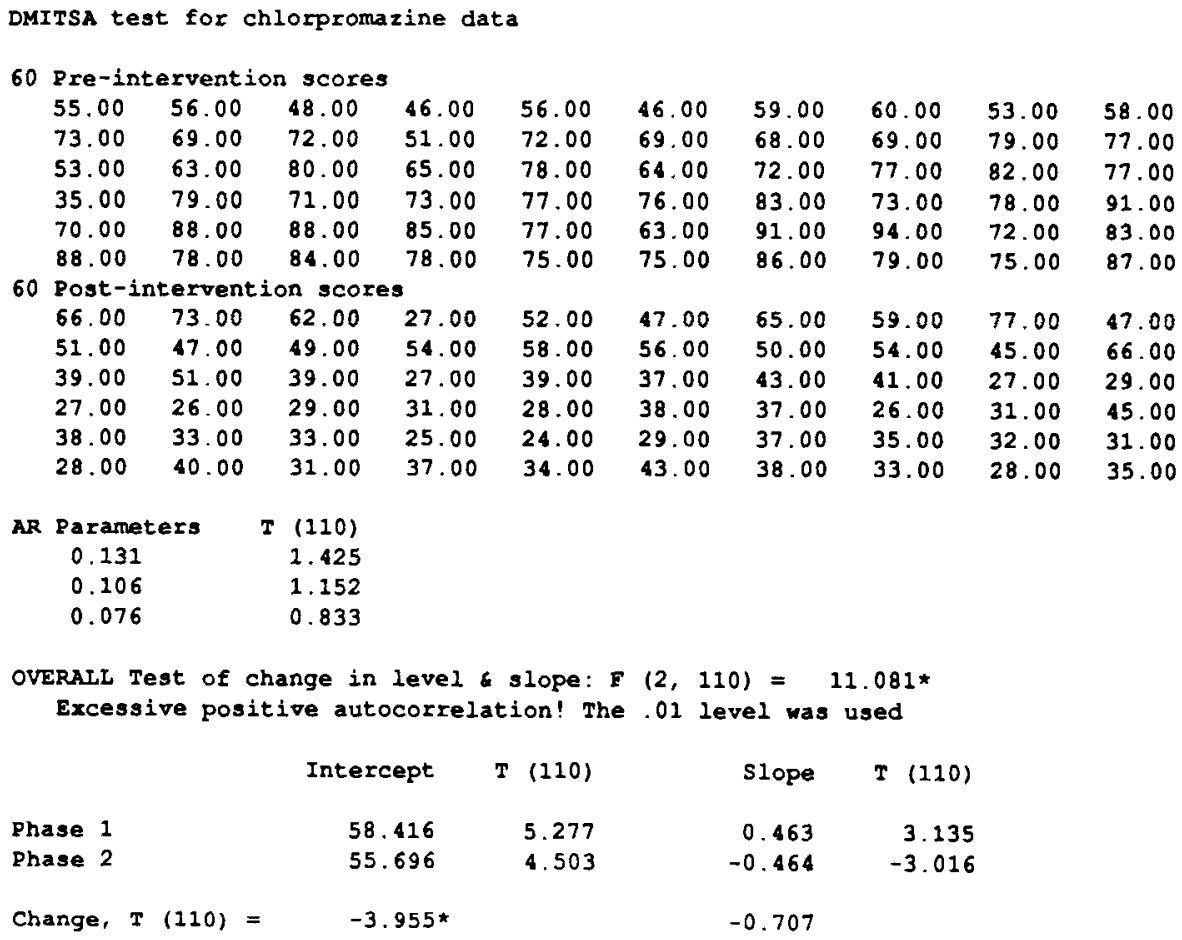

Figure 4. File output (CHLOR1.OUT) for a DMITSA analysis of CHLOR1.DAT. 
played on the screen, except that each intercept and slope has an associated $t$ value for the null hypothesis that the intercept or slope is zero.

\section{Availability}

A copy-protected version of the program (on a 5.25-in. 360K DSDD diskette for the IBM PC family of computers) and a user manual are available from Christopher Sharpley, Faculty of Education, Monash University, VIC 3168 , Australia. The single-user cost is US $\$ 150$, but multiuser licenses can be negotiated. Checks should be made payable to Behavioural Computing.

\section{REFERENCES}

Crosbie, J. (1987). The inability of the binomial test to control Type I error with single-subject data. Behavioral Assessment, 9, 141-146.

Glass, G. V., Willson, V. L., \& Gottman, J. M. (1975). Design and analysis of time-series experiments. Boulder, $\mathrm{CO}$ : University of Colorado Press.

GotTman, J. M. (1981). Time-series analysis: A comprehensive introduction for social scientists. London: Cambridge University Press.

Hartmann, D. P., Gottman, J. M., Jones, R. R., Gardner, W., KAZdin, A. E., \& VAUGHT, R. (1980). Interrupted time-series analysis and its application to behavioral data. Journal of Applied Behavior Analysis, 13, 543-559.

Williams, E. A., GotTman, J. M. (1982). A user's guide to the Gottman-Williams time-series analysis programs for social scientists. London: Cambridge University Press.

(Manuscript received June 13, 1989;

accepted for publication September 6, 1989.) 